\title{
Clinical laboratory practices for detection and reporting of Cryptosporidium in community cases of diarrhoea in the United Kingdom, 2008
}

R M Chalmers (rachel.chalmers@wales.nhs.uk) ${ }^{1}$, B Campbell ${ }^{2}$, N Crouch $^{1}$, A P Davies ${ }^{2}$

1. Cryptosporidium Reference Unit, Public Health Wales Microbiology, Singleton Hospital, Swansea, Wales, United Kingdom

2. School of Medicine, Swansea University, Singleton Park, Swansea, Wales, United Kingdom

To identify procedures employed by publicly funded clinical diagnostic laboratories in the United Kingdom (UK) for the detection of Cryptosporidium in community cases of diarrhoea, a telephone survey was conducted between August 2008 and January 2009 of all such laboratories that test stools from communitybased patients. All 200 laboratories responded: 145 (72.5\%) tested all stool samples for Cryptosporidium, while $55(27.5 \%)$ applied selection criteria. There were country and regional differences in the proportion of laboratories selectively testing stools, which were significantly correlated with Cryptosporidium report rates to national surveillance (Spearman's rank correlation coefficient $\left(r_{s}\right)=0.61$, degrees of freedom $(d f)=11, p=0.03)$. Understanding of laboratory practice is fundamental to interpreting trends in surveillance data, estimating disease burden and identifying outbreaks, as well as providing important background information against which changes and effects of new public health regulations can be measured.

\section{Introduction}

Cryptosporidium is a protozoan parasite that infects a broad host range, causes gastrointestinal illness in humans and young animals and constitutes a significant risk to public health, especially among young children and immunocompromised patients with specific T-cell deficiencies [1]. Transmission is by the faecaloral route. Particular challenges to control arise from the ability of the transmissive stage, the oocyst, to survive conventional water treatment and its resistance to chlorine disinfection, resulting in waterborne disease in developed and developing countries. Laboratory testing is necessary for differential diagnosis of gastroenteritis and implementation of appropriate control measures, and requires submission of a stool sample. However, clinicians and public health professionals should be aware that not all laboratories test all stools for Cryptosporidium and that Cryoptosporidium testing is not necessarily specified in a test request for ova, cysts and parasites.
In the early 1990s, following a waterborne outbreak of cryptosporidiosis in Swindon, Wiltshire, and neighbouring parts of Oxfordshire, England [2], an expert group was established by the then Departments of Health and Environment to advise the United Kingdom (UK) Government on the significance of the presence of Cryptosporidium in water supplies. One of the recommendations of the ensuing report was that there should be a review and standardisation of clinical laboratory policies for examining faecal samples for Cryptosporidium [3]. Evidence from a two-year prospective study in 16 laboratories where all stools were tested for Cryptosporidium showed that $60 \%$ of positive stools were from children up to 15 years of age and that $90 \%$ were from people under 45 years of age [4]. Thus a joint working group recommended that, where possible, all specimens from symptomatic individuals should be tested, but where this was not feasible, children and adults up to 45 years should be tested [5]. As a minimum requirement, all children up to and including 15 years of age should be tested. Recommended methods were microscopic examination of faecal smears stained with auramine phenol (AP) or a modified Ziehl-Neelsen's (mZN) stain [6]. In the UK, stools are submitted fresh, without formalin, so concentration is not required routinely prior to staining. Where concentration is deemed necessary, modified methods should be used to minimise oocyst losses and prevent interference with the adhesion of oocysts to slides and with staining [6]. Examination of stained smears is required because the oocysts are too small $(4-6 \mu \mathrm{m})$ for accurate identification by unstained observation of wet faecal films [6]. The national standard method of the Health Protection Agency (HPA) currently recommends that all samples from symptomatic individuals should be tested for Cryptosporidium [7] by microscopy using AP or mZN staining, with confirmation by staining a new smear using mZN stain [8]. The use of enzyme immunoassays (EIAs) is acknowledged but is not specified as a national standard method. Despite these recommendations, a variable pattern of testing has been identified previously [9-11]. In 2006 we sur- 
veyed 169 laboratories in the UK: 36 (21.3\%) applied selection criteria (unpublished data).

Cryptosporidium is included in Directive 2003/99/ EC the European Parliament and the Council of the European Union [12], which directs monitoring and data collection of zoonoses and zoonotic agents. Cryptosporidiosis is therefore a notifiable disease within the European Union and laboratory-confirmed case data are collected through the European Surveillance System (TESSy). However, the disease is statutorily notifiable in only some European countries (e.g. Germany, Ireland and Sweden). In 2007, only 10 of the 30 countries in TESSy reported any cryptosporidiosis cases [13] and it is likely that there are substantial differences in ascertainment between countries [14]. Ireland reported the highest notification rates in 2007 (14 per 100,000 population), followed by the UK (six per 100,000 population). In the UK national surveillance has been based on voluntary, passive laboratory reporting of diagnosed cases (either by paper reports or through interconnected computer database modules to the health protection agencies). Prior to 2010 cryptosporidiosis was only statutorily notifiable as part of food poisoning notifications. However, under new regulations taking forward the modernisation of health protection law, the Health Protection (Notification) Regulations 2010, Cryptosporidium is included in the list of causative agents known as Schedule 2 and so detection of the parasite became notifiable by UK laboratories in October 2010, except in Scotland, where Cryptosporidium became notifiable on 1 January 2010 under the Public Health etc. (Scotland) Act 2008.
Baseline knowledge of the policies and practices for detecting and reporting Cryptosporidium is essential for assessing disease burden, comparing surveillance data, identifying outbreaks, and implementing and monitoring interventions. To establish clinical laboratory procedures prior to changes in notification requirements, and to identify changes since the previous surveys, we surveyed between August 2008 and January 2009 the policies of publicly funded laboratories throughout the UK concerning Cryptosporidium testing and reporting.

\section{Methods}

All 200 publicly funded clinical laboratories that test stools from community-based patients in the UK were contacted by telephone between 20 August 2008 and 27 January 2009. One person (B. Campbell) interviewed the consultant microbiologist, laboratory manager or lead biomedical scientist using a structured questionnaire. The questions were about selection criteria for testing stools for Cryptosporidium, diagnostic tests and reporting results, and referred to community cases of diarrhoea.

Data were recorded and analysed in Microsoft Excel. Regional and national numbers of reported cryptosporidiosis cases were obtained from the websites of the HPA (for England and Wales) [15], the Communicable Disease Surveillance Centre (Northern Ireland) [16] and Health Protection Scotland [17]. Report rates per 100,000 population were calculated using mid-year population estimates for 2008 [18] as denominator and were compared with the proportion of laboratories testing all stools. Spearman's rank correlation coefficient

\section{TABLE}

Laboratory testing of community cases of diarrhoea for Cryptosporidium and reports to national surveillance in the United Kingdom, 2008

\begin{tabular}{|c|c|c|}
\hline $\begin{array}{l}\text { Country or Government office } \\
\text { region (England only) }\end{array}$ & $\begin{array}{c}\text { Number of laboratories } \\
\text { testing all stools for Cryptosporidium/number of } \\
\text { laboratories (\%) }\end{array}$ & $\begin{array}{c}\text { Number of Cryptosporidium reports to national surveillance } \\
\text { (rate per 100,000 population) }\end{array}$ \\
\hline England & $100 / 154(65)$ & $3,885(7.5)$ \\
\hline East & $12 / 18(67)$ & $469(8.2)$ \\
\hline East Midlands & $7 / 8(88)$ & $547(12.3)$ \\
\hline London & $7 / 23(30)$ & $197(2.6)$ \\
\hline North East & $3 / 9(33)$ & $193(7.5)$ \\
\hline North West & $24 / 27$ (89) & $673(9.8)$ \\
\hline South East & $11 / 22(50)$ & $467(5.6)$ \\
\hline South West & $10 / 15(67)$ & $458(8.8)$ \\
\hline West Midlands & $14 / 17(83)$ & $383(7.1)$ \\
\hline $\begin{array}{l}\text { Yorkshire and the } \\
\text { Humber }\end{array}$ & $12 / 15(80)$ & $498(9.6)$ \\
\hline Wales & $14 / 14(100)$ & $239(8.0)$ \\
\hline Northern Ireland & $5 / 6(83)$ & $119(6.7)$ \\
\hline Scotland & $26 / 26(100)$ & $613(11.9)$ \\
\hline Total & $145 / 200(73)$ & $4,856(7.9)$ \\
\hline
\end{tabular}

a Data sources: Health Protection Agency Centre for Infections (England and Wales) [15]; Public Health Agency Communicable Disease Surveillance Centre (Northern Ireland) [16] and Health Protection Scotland [17]. 
$\left(r_{s}\right)$ was obtained by applying the formula for Pearson's $r$ to the ranks of the two variables.

\section{Results}

Of the 200 laboratories surveyed, all confirmed that they tested specimens from community cases of diarrhoea. Of these, 154 laboratories were in England, 14 in Wales, 26 in Scotland, and six in Northern Ireland. A total of $145(72.5 \%)$ tested all stool samples from community cases of diarrhoea for Cryptosporidium while 55 $(27.5 \%)$ applied selection criteria. There were country and regional differences in the proportion of laboratories selectively testing stools: in Scotland and Wales all stools were tested, in Northern Ireland only one laboratory applied selection criteria, but in London, England, only seven of 23 laboratories tested all stools (Table).

There was a positive relationship between Cryptosporidium reports to national surveillance and completeness of laboratory testing at the regional level; the two were significantly correlated $\left(r_{s}=0.61\right.$, degrees of freedom $(d f)=11, p=0.03$ ) (Figure).

Of the 55 laboratories that selectively tested stools for Cryptosporidium, one or more of the following criteria were applied: age $(n=38)$, immune status $(n=36)$, stool consistency $(n=37)$, duration of diarrhoea $(n=3)$, overseas travel $(n=22)$, farm visit or animal contact $(n=17)$, clinician's request $(n=13)$ and during an outbreak $(n=3)$. The 38 laboratories that selected specimens according to age used the following categories: $>6$ months $(n=1)$, 6 months to 60 years $(n=1),<2$ years $(n=1),<5$ years $(n=3),<6$ years $(n=1),<8$ years $(n=1),<9$ years $(n=1),<10$ years $(n=4),<11$ years $(n=1),<12$ years $(n=1),<14$ years $(n=2),<15$ years $(n=6),<16$ years $(n=9),<45$ years $(n=5)$ and $<50$ years $(n=1)$.

The diagnostic methods used were mainly staining of faecal smears, either by an mZN stain $(n=44,21.9 \%$ laboratories) or AP stain with a variety of counterstains

\section{FIGURE}

Relationship between regional rate of Cryptosporidium reports and completeness of laboratory testing in each region, United Kingdom, 2008

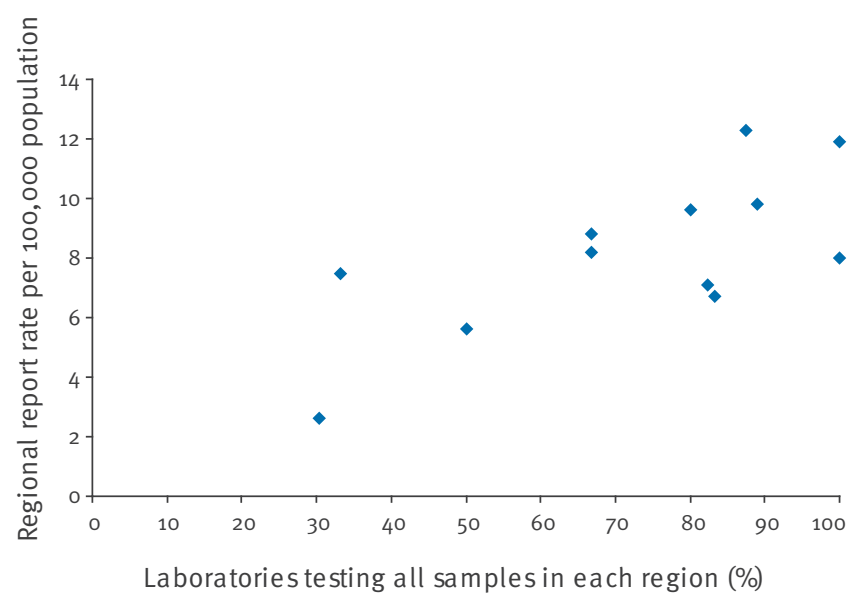

( $n=151,75.5 \%$ laboratories). Of the laboratories using AP staining, 61 (40.4\%) confirmed the finding by mZN staining. One laboratory also used immunofluorescence microscopy to resolve equivocal results. Parasite concentration methods were used prior to staining by seven laboratories; six used commercially available faecal parasite concentrators and one an in-house diethyl ether method. In the UK, stools are submitted fresh and so concentration is not usually required.

Five laboratories used commercially available EIAs as the primary test. Two of these were Cryptosporidiumonly assays and three were combination assays for the simultaneous detection of Cryptosporidium and Giardia. All five laboratories using EIAs confirmed findings by a second method; the Cryptosporidium-only assays were confirmed by staining and microscopy and the combination assays with either staining and microscopy or commercially available Cryptosporidiumspecific immunochromatographic tests.

Reporting for surveillance purposes was undertaken by 190 (95\%) laboratories. Reporting to local authorities was also undertaken by 190 ( $95 \%$ ) laboratories (not the same 190).

\section{Discussion}

Current UK guidance on laboratory testing is that stools from all community cases of diarrhoea should be tested for Cryptosporidium [7]. Although this was achieved by almost three quarters of laboratories nationally, there were large differences between the countries and regions and this may have an effect on the assessment of the burden of illness and ability to monitor outbreaks as well as measure changes in the number of reported cryptosporidiosis cases and report rates. It is of particular concern that 23 laboratories did not meet the minimum requirement of testing all children up to and including 15 years of age. Furthermore, where age criteria were applied these varied enormously with often no apparent rationale for the age cut-offs, leading to further inconsistencies in the data. The effect of age policy on case age distribution has been reported previously [11]. The value of testing adults, particularly up to age 45 years, has been demonstrated [4] and their inclusion in testing plays a key role in the ability to detect outbreaks, particularly those linked to drinking water, which affect all ages. It is well documented that one of the features of waterborne outbreaks is an excess of adult cases [19]. The use of inappropriate criteria has also been observed, especially submitted stool consistency, which is an unreliable predictor of Cryptosporidium positivity [20]. We discussed our findings with the regional microbiology network. The data demonstrate to clinicians that, even in the UK where testing is widespread, if cryptosporidiosis is suspected clinically then Cryptosporidium should be specified on the request form to ensure appropriate testing.

Despite some inadequacies, most stool samples are tested for Cryptosporidium in most regions of the UK 
and this appears to be a relatively stable situation, with some notable exceptions. Overall, there has been little change between 2006 and 2008 with respect to the proportion of laboratories testing all samples. New developments in streamlining of laboratory testing are now available in the form of automated microscope slide staining and reliable alternatives to microscopy are provided by second-generation EIAs. These, particularly when coupled with automated processing and reading devices, simplify laboratory testing and assist in the standardisation of laboratory methods. Additional benefits have been demonstrated from the application of combination assays - for example, improved ascertainment of Giardia, particularly in groups not considered traditionally for testing [21]. Since our survey was completed, we are aware of at least two more laboratories that have replaced microscopy with EIA for Cryptosporidium and Giardia detection and more laboratories are using immunochromatographic assays. Alternatively, multiplex polymerase chain reaction (PCR) assays for routine diagnosis of various combinations of gastrointestinal pathogens including Cryptosporidium offer streamlined and modernised laboratory testing, and are routinely used in the Netherlands [22] and are under investigation in the UK.

Although an association cannot be proven by such analysis, there was a correlation between increased annual reporting rates and the proportion of laboratories testing all stools at the regional level. The analysis was imperfect because, while surveillance data may include reports from inpatients, only data related to testing community samples were used. However, we consider this would not lead to much discrepancy, as the vast majority of cases are diagnosed from community settings. Furthermore, there are other possible reasons for genuine regional or local variation in the numbers of reported cryptosporidiosis cases, such as exposure, environmental and socio-economic factors $[23,24]$. Despite this, the trend for correlation between regional reporting and testing rates is notable, as shown in the Figure. The higher cryptosporidiosis rates reported by the UK to TESSy, compared with most other European countries, are undoubtedly influenced in part by differences in approaches to laboratory testing and data collection. Standardised laboratory testing and reporting of pathogens in the European setting is important in minimising discrepancies, ensuring comparability of surveillance data and enabling rapid public health action in the event of international incidents and outbreaks [12].

A high proportion of laboratories already report cryptosporidiosis cases to local authorities for further investigation and to surveillance systems in the UK. Although statutory notification will ensure high ascertainment where all samples are tested for Cryptosporidium, the requirements of the new Health Protection (Notification) Regulations do not extend to mandatory testing, and there will still be an effect on the reported incidence of cryptosporidiosis. This has been observed in Ireland, where Cryptosporidium became statutorily notifiable in $\mathbf{2 0 0 4}$, but a large number of laboratories have opted to apply an age threshold as a selection criterion [25]. The interpretation of routine laboratory surveillance data is difficult without standardised policies, and without these, comparisons at regional, national and international levels are hampered [14]. Cryptosporidiosis remains an underreported disease despite the increase in testing in countries such as the UK. Understanding of laboratory practice is fundamental to understanding trends in surveillance data, estimating disease burden and identifying outbreaks.

\section{Acknowledgements}

We wish to thank all the laboratory staff who provided the information on which this report is based.

\section{References}

1. Hunter PR, Nichols G. Epidemiology and clinical features of Cryptosporidium infection in immunocompromised patients. Clin Microbiol Rev. 2002;15(1):45-54.

2. Richardson AJ, Frankenberg RA, Buck AC, Selkon JB, Colbourne JS, Parsons JW, et al. An outbreak of waterborne cryptosporidiosis in Swindon and Oxfordshire. Epidemiol Infect. 1991;107(3):485-95.

3. Group of Experts. Cryptosporidium in water supplies. Report of the Group of Experts. Chairman Sir John Badenoch. London: Her Majesty's Stationery Office; 1990.

4. Palmer SR, Biffin AH. Public Health Laboratory Service Study Group. Cryptosporidiosis in England and Wales: prevalence and clinical and epidemiological features. BMJ. 990;300(6727):774-7.

5. Casemore DP, Roberts C. Guidelines for screening for Cryptosporidium in stools: report by a joint working group. Clin Pathol. 1993;46(1):2-4.

6. Casemore DP. ACP Broadsheet 128: June 1991. Laboratory methods for diagnosing cryptosporidiosis. J Clin Pathol. 1991;44(6):445-51.

7. Health Protection Agency (HPA). Investigation of specimens other than blood for parasites. National Standard Method BSOP 31. Issue 3. London: HPA; 2008. Available from: http:// www.hpa-standardmethods.org.uk/documents/bsop/pdf/ bsop31.pdf

8. Health Protection Agency (HPA). Staining procedures. National Standard Method BSOP TP 39. Issue 1. London: HPA; 2007. Available from: http://www.hpa-standardmethods.org.uk/ documents/bsopTP/pdf/bsoptp39.pdf

9. Chalmers RM, Hughes S, Thomas AL, Woodhouse S, Thomas PD, Hunter P. Laboratory ascertainment of Cryptosporidium and local authority public health policies for the investigation of sporadic cases of cryptosporidiosis in two regions of the United Kingdom. Commun Dis Public Health. 2002;5(2):114-8.

10. Crook P, Mayon-White R, Reacher M. Enhancing surveillance of cryptosporidiosis: test all faecal specimens from children. Commun Dis Public Health. 2002;5(2):112-3.

11. Campbell DM, Flannagan PA. Laboratory examination for Cryptosporidium spp. in Scotland. J Clin Pathol. 1992;45(10):914-6.

12. European Parliament and the Council of the European Union. Directive 2003/99/EC of the European Parliament and of the Council of 17 November 2003 on the monitoring of zoonoses and zoonotic agents, amending Council Decision 90/424/EEC and repealing Council Directive 92/117/EEC. Official Journal of the European Union. Luxembourg: Publications Office of the European Union. 12.12.2003: L 325/31. Available from: http:// eur-lex.europa.eu/LexUriServ/LexUriServ.do?uri=0J:L:2003:32 5:0031:0040:EN:PDF

13. European Centre for Disease Prevention and Control (ECDC). Annual epidemiological report on communicable diseases in Europe 2009. Stockholm: ECDC; 2009 . Available from: http:// www.ecdc.europa.eu/en/publications/Publications/0910_ SUR_Annual_Epidemiological_Report_on_Communicable Diseases_in Europe.pdf 
14. Semenza J, Nichols G. Cryptosporidiosis surveillance and water-borne outbreaks in Europe. Euro Surveill. 2007;12(5). pii: 711. Available from: http://www.eurosurveillance.org/ ViewArticle.aspx?Articleld $=711$

15. Health Protection Agency (HPA). All identifications of cryptosporidium, 1989-2008. Available from: http://www.hpa.org.uk/Topics/InfectiousDiseases/ InfectionsAZ/Cryptosporidium/EpidemiologicalData// cryptoallcases19892008/

16. Communicable Disease Surveillance Centre Northern Ireland (CDSC(NI)). Laboratory reports of Cryptosporidium sp (all specimen types). 1995-2009. Belfast: CDSC(NI). Available from: http://www.cdscni.org.uk/surveillance/Gastro/ Cryptosporidium_sp.htm

17. Health Protection Scotland (HPS). Gastro-intestinal and foodborne infections: laboratory reports of viral and protozoal infections reported to HPS in 2008. HPS weekly report. 11 February 2009. Volume 43, No. 2009/06. p. 67-9. Available from: http://www.documents.hps.scot.nhs.uk/ewr/ pdf2009/0906.pdf

18. Office for National Statistics (ONS). Population estimates for UK, England and Wales, Scotland and Northern Ireland current datasets. ONS: Titchfield, Fareham. [Accesssed 4 Mar 2010]. Available from: http://www.statistics.gov.uk/statbase/ product.asp?vlnk=15106

19. Meinhardt P, Casemore DP, Miller KB. Epidemiologic aspects of human cryptosporidiosis and the role of waterborne transmission. Epidemiologic Rev. 1996;18(2):118-36.

20. Casemore DP, Armstrong M, Sands RL. Laboratory diagnosis of cryptosporidiosis. J Clin Pathol. 1985;38(12):1337-41.

21. Ellam H, Verlander NQ, Lamden K, Cheesbrough JS, Durband CA, James S. Surveillance of giardiasis in Northwest England 1996-2006: impact of an enzyme immunoassay test. Euro Surveill. 2008;13(37). pii: 18977. Available from: http://www. eurosurveillance.org/ViewArticle.aspx?Articleld=18977

22. Bruijnesteijn van Coppenraet LE, Wallinga JA, Ruijs GJ, Bruins MJ, Verweij JJ. Parasitological diagnosis combining an internally controlled real-time PCR assay for the detection of four protozoa in stool samples with a testing algorithm for microscopy. Clin Microbiol Infect. 2009;15(9):869-74.

23. Hunter PR, Hughes LS, Woodhouse S, Syed Q, Verlander N, Chalmers RM, et al. Sporadic cryptosporidiosis case-contro study with genotyping. Emerg Infect Dis. 2004;10(7):1241-9.

24. Lake IR, Harrison FCD, Chalmers RM, Bentham G, Nichols G, Hunter PR, et al. Case-control study of environmental and social factors influencing cryptosporidiosis. Eur J Epidemiol. 2007;22(11):805-11.

25. Garvey P, McKeown P. Epidemiology of human cryptosporidiosis in Ireland, 2004-2006: analysis of national notification data. Euro Surveill. 2009;14(8). pii: 19128. Available from: http://www.eurosurveillance.org/ViewArticle. aspx?Articleld $=19128$ 